\title{
Optimization of Threshold based Rate Adaptation Technique in Wireless Network
}

\author{
Lily Mishra \\ Postgraduate Student \\ Department of Electronics and Telecommunication \\ D.J.Sanghvi college of Engineering, \\ Mumbai, India
}

\author{
M.H.Patwardhan \\ Assistant Professor \\ Department of Electronics and Telecommunication \\ D.J.Sanghvi college of Engineering, \\ Mumbai, India
}

\begin{abstract}
Adaptive Modulation and Coding technique has emerged as a bandwidth efficient transmission scheme. It is motivated by its ability to improve spectral efficiency by adapting the transmission rates to the variations in channel signal-to-noise ratio. The implementation of apt rate adaptation algorithms based on either signal to noise ratio or successful packet transmission has been the crucial factor in Adaptive Modulation and Coding. This work focuses upon enhancing the data rate while implementing Adaptive Modulation and coding by modifying the rate adaptation algorithm in the context of IEEE 802.11 WLAN network. The signal to noise ratio threshold based rate adaptation algorithm was modified using MATLAB/SIMULINK. The said modification in the algorithm without disturbing the signal-to-noise ratio threshold values resulted into the enhancement of data rates. It also demonstrated that in the varying channel conditions, the modified algorithm would be able to minimize the number of transitions needed to achieve a particular mode of adaptive Modulation and Coding.
\end{abstract}

\section{Keywords}

Adaptive Modulation and Coding (AMC), Rate adaptation, signal-to-noise ratio, threshold value.

\section{INTRODUCTION}

The IEEE 802.11 standards support multiple bit rates at the physical layer so that stations can maximize the system performance by controlling the transmission rate responding to the conditions of underlying time-varying channels. The process is basically known as link adaptation which is commonly known by the name Adaptive Modulation and Coding (AMC) as well. With the technological advancement, the demand for bandwidth has increased many fold. Especially in wireless communication where bandwidth is a shared resource, spectrally efficient transmission schemes have always been in demand. AMC could be implemented by varying different parameters known as degrees of freedom and varying the data rate is one of the popular varieties known as Rate Adaptation. A number of Rate Adaptation schemes using Rate-adaptive Acknowledgment (RARA) [2], Rate Adaptation in Mobile Environments (RAM) [3], Rate Adaptation using Coherent time (REACT) [4] are used to implement AMC in IEEE 802.11 environment.

\section{RELATED WORK}

\subsection{Rate Adaptation}

Rate adaptation is the process of dynamically switching data rates to match the channel conditions, with the goal of selecting the rate that will give the optimum throughput for the given channel conditions. Rate Adaptation is the heart of AMC. It helps choose the best rate for the current channel condition. The aim is to maximize the throughput by exploiting the multiple transmission rates available for 802.11 devices and varying their transmission rates dynamically to the time-varying and location dependent wireless channel conditions.

\subsection{Threshold-based Rate Adaptation}

The threshold-based rate adaptation algorithm is a special case of rate adaptation algorithm. In [5], it has been first investigated via a reverse engineering approach where the implicit objective function is revealed. Then, a threshold optimization algorithm is proposed which can dynamically adjust the up/down thresholds and converge to the stochastic optimum solution in arbitrary stationary random channel environment. In the same paper, two categories of rate adaptation are defined namely open loop and closed loop. Most commercialized IEEE products follow this up/down scheme. The paper has focused upon the open-loop rate adaptation algorithms due to the practical merits.

In our work, we have focused upon a threshold based rate adaptive algorithm which uses the channel condition known by measuring the SNR value according to which the bit rate of the system is decided. The bit rate corresponds to which mode gets selected depending upon the SNR value. The mode is restricted by the up and down threshold values of the SNR. However, we have tried to enhance the bit rate keeping the up/down threshold values to be fixed for a particular mode. The set of data rates, modulation and coding rates used are as per IEEE specifications [6]. The table no.1 below shows the same:-

Table 1: Various Modes

\begin{tabular}{|c|c|c|}
\hline Modulation & Coding Rate (R) & Data Rate( Mbps) \\
\hline BPSK & $1 / 2$ & 6 \\
\hline BPSK & $3 / 4$ & 9 \\
\hline QPSK & $1 / 2$ & 12 \\
\hline QPSK & $3 / 4$ & 18 \\
\hline $16-Q A M$ & $1 / 2$ & 24 \\
\hline $16-Q A M$ & $3 / 4$ & 36 \\
\hline $64-Q A M$ & $2 / 3$ & 48 \\
\hline $64-Q A M$ & $3 / 4$ & 54 \\
\hline
\end{tabular}




\section{Flowchart of AMC Algorithm}

The logic of the flowchart is explained as below:-

1) The eight different modes are initialized first and SNR is fed as the input.

2) It is compared with the SNR up threshold of the $1^{\text {st }}$ mode, if it is greater, then the mode is increased to the next higher mode i.e, mode +1 . If it is not then the input SNR value is compared with the SNR down threshold value.

3) If the input is lesser than or equal to the SNR down threshold then mode is decremented. Otherwise the same mode is selected.

4) Corresponding bit rate is achieved and next value of SNR as input is fetched.

Fig.1. shows the flowchart for a threshold based rate adaptation algorithm which has been used in the AMC model implemented as the core of the working of the system.

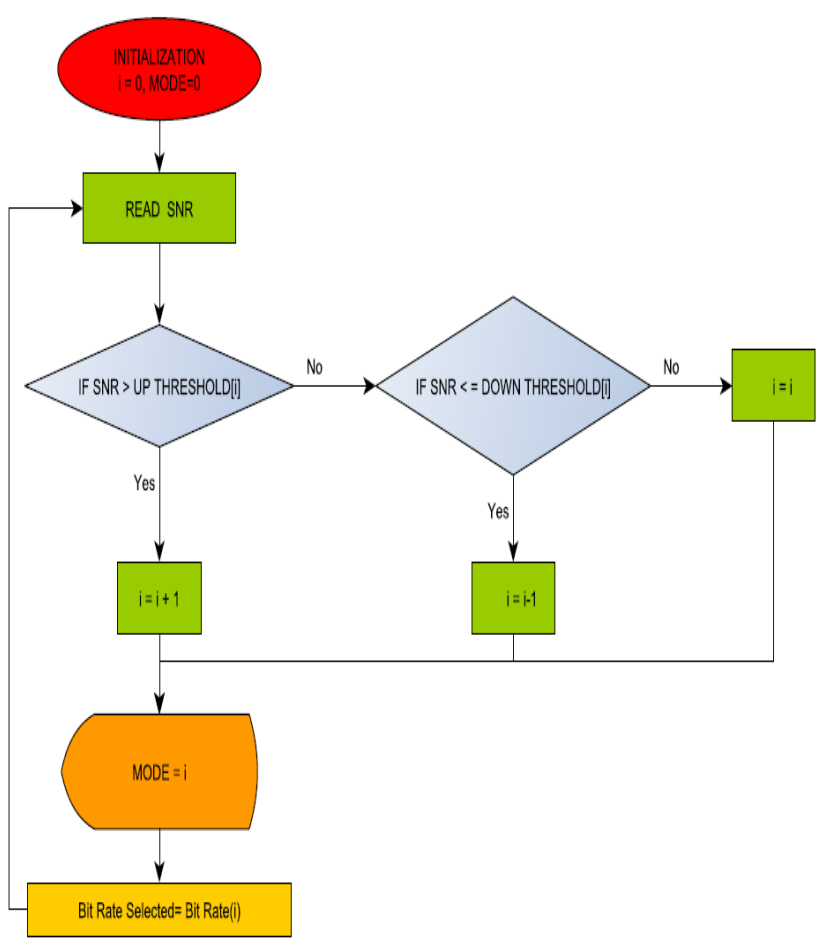

Fig.1. Flowchart of Original Threshold Based Rate Adaptation Algorithm

\section{OVERVIEW OF THE RESEARCH PROBLEM AND THE PROPOSED SOLUTION APPROACH}

A number of different protocols, schemes and algorithms were studied which were used to implement the process of Adaptive Modulation and Coding. The classification scheme and categorization methods from the relevant literature indicated that threshold based rate adaptation algorithms were the most frequently used for IEEE 802.11 standard. However, these algorithms differed in the way the process of adaptation was executed.

The issues of major concern in the implementation of AMC in wireless environment have been, apt selection of modulation scheme and coding rate (i.e. the mode of AMC), the average data rate achievable in the network with AMC implementation, and the dynamicity of the rate adaptation, especially in the presence of fast fading environment. Thus, ideally the rate adaptation algorithm implemented should be able to maximize the data rate, at faster pace and all this without compromising the quality parameters of the link in the network. The enhancement of data rate at a faster pace with the same set of threshold SNR values remained an issue which has been addressed in our work to achieve optimization in AMC implementation. The following have been the major implementation steps of the work.

- Development/ Adaptation of the basic SIMULINK model for IEEE 802.11

- Verification of implementation AMC scheme in the above mentioned model and compilation of the relevant results.

- Logical development of the proposed modified Rate Adaptation algorithm

- Coding and execution of the proposed modified Rate Adaptation algorithm

- Comparing the performance of the proposed modified Rate Adaptation algorithm with the original Rate Adaptation algorithm

\section{MODIFYING THE RATE ADAPTATION ALGORITHM}

In order to achieve improvement in the data rate at a faster pace, the existing original algorithms has been modified and its revised version has been proposed. The proposed algorithm has been implemented in MATLAB which has the potential to get interfaced with the SIMULINK modelthe proposed modification is as given below:-

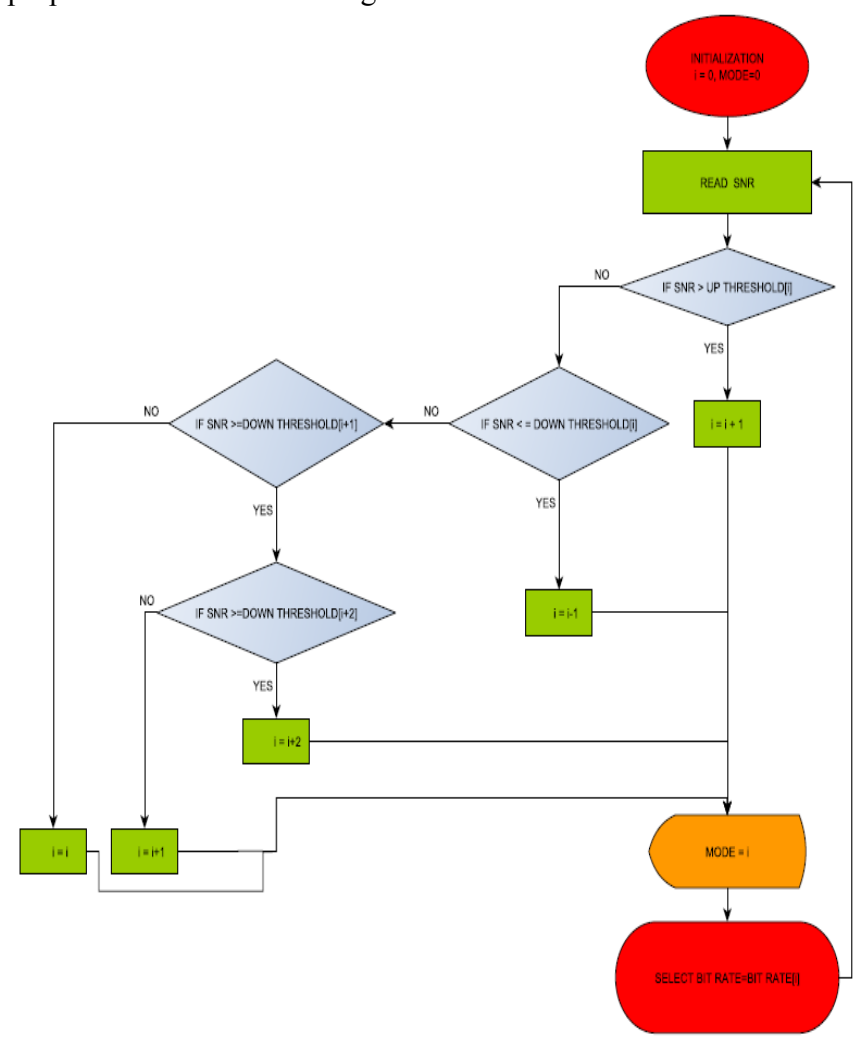

Fig.2 Flowchart of Modified Algorithm 
The logic of the flowchart is explained as below:-

1) The eight different modes are initialized first and SNR is fed as the input.

2) It is compared with the SNR up threshold of the $1^{\text {st }}$ mode, if it is greater, then the mode is increased to the next higher mode i.e, mode +1 . If it is not then the input SNR value is compared with the SNR down threshold value.

3) If the input is greater than the SNR down threshold then mode is decremented. However if it is found to be lesser than or equal to the down threshold then it is compared with the down threshold of the next higher mode and the process repeats and repeats till it gets till suitable mode gets selected.

Accordingly the mode is incremented or decremented and bit rate corresponding to it is achieved. Then the next value of SNR as input is fetched.

\section{RESULTS}

The performance of the modified threshold based rate adaptation scheme was analyzed as per themethodology explained here. The important parameters to observe for assessing the performance of the rate adaptation algorithms are:

1) Data rate enhancement: As SNR increases, it is expected that the higher modes will get allotted by the algorithm leading to the enhanced data rate.

2) Time to acquire a particular mode: When higher data rates are being allotted, how soon the link adapts or allots a particular higher mode can be analyzed on the basis of number of transition that algorithm goes through before getting allotted the final higher mode.

\subsection{Comparison of the data rate achieved:}

The following methodology was adapted to compare the data rate enhancement performance of the original and the modified algorithm

a) Generate a set of thousand randomized SNR values and feed that as an input to the algorithm

b) Observe and find out the modes allotment done as per the algorithm being implemented

c) Identify the data rates allotted as per the allotment of the mode.

d) Step a) to c) will form one complete iteration.

e) Repeat the iteration (i.e. step a) to c))for number of times

f) Find out the average data rate after completing all the iterations.

Table no.1 shows the number of times a particular mode got allotted in each iteration when thousand randomize values of SNR were fed in to the original algorithm. The next table i.e, table no. 2 shows the number of times a particular mode got allotted in each iteration when thousand randomized values of SNR were fed in to the modified algorithm. The average of a particular mode allotted by implementing both the algorithms (original and modified) are provided in table no.3.
Table 1:Mode allocation data of original algorithm

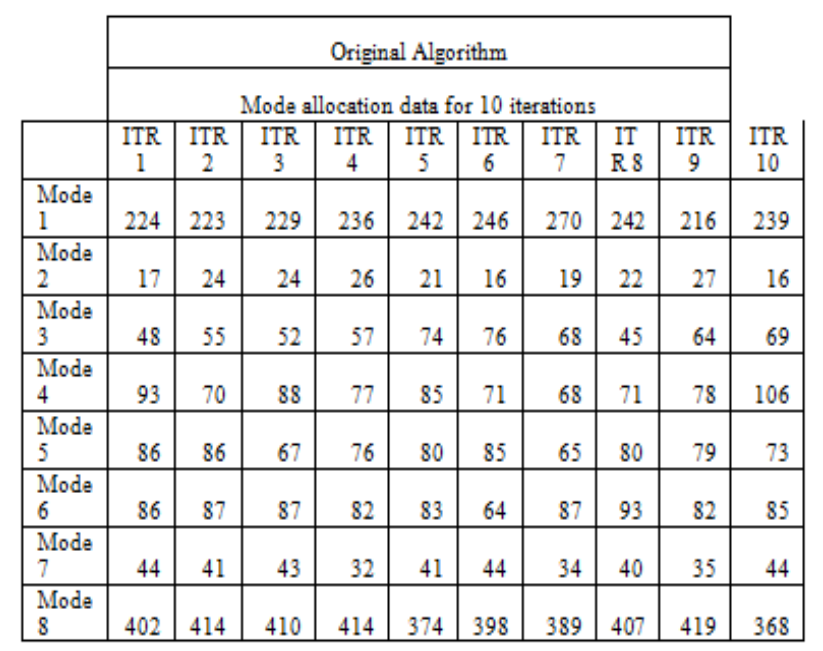

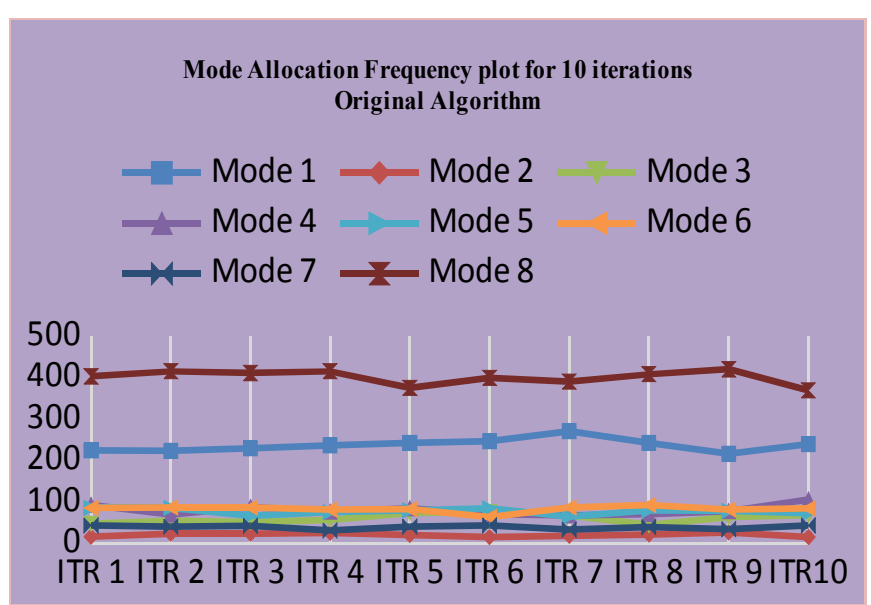

Fig.3. Mode Allocation Frequency Plot of Original Algorithm

Table 2: Mode allocation data of modified algorithm

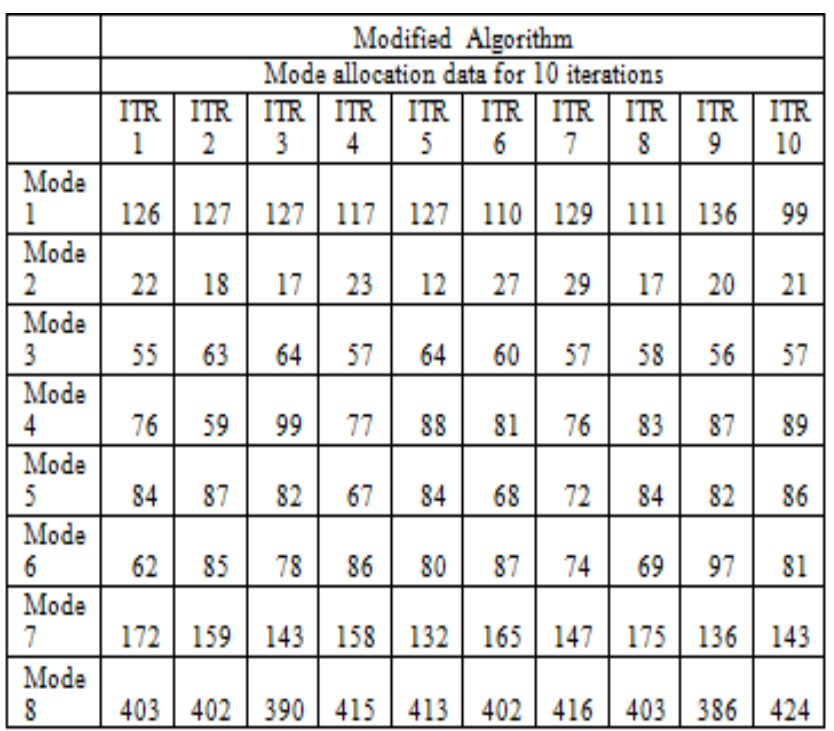




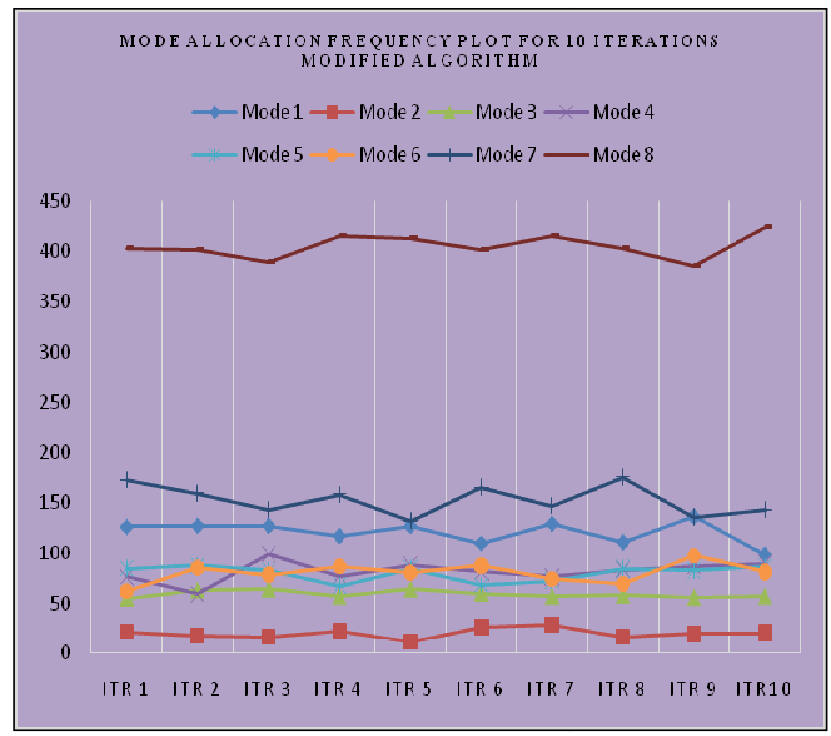

Fig.4. Mode Allocation Frequency Plot of Modified Algorithm

Table 3: Mode allocation comparison: Original v/s Modified Algorithm

\begin{tabular}{|l|c|c|}
\hline \multicolumn{3}{|c|}{ Mode allocation comparison } \\
\hline & No. of times modes allotted \\
\hline Original Algorithm & New Algorithm \\
\hline Mode 1 & 237 & 121 \\
\hline Mode 2 & 21 & 21 \\
\hline Mode 4 & 61 & 59 \\
\hline Mode 5 & $\mathbf{8 1}$ & 82 \\
\hline Mode 6 & 84 & 80 \\
\hline Mode 7 & 40 & 80 \\
\hline Mode 8 & 400 & 153 \\
\hline
\end{tabular}

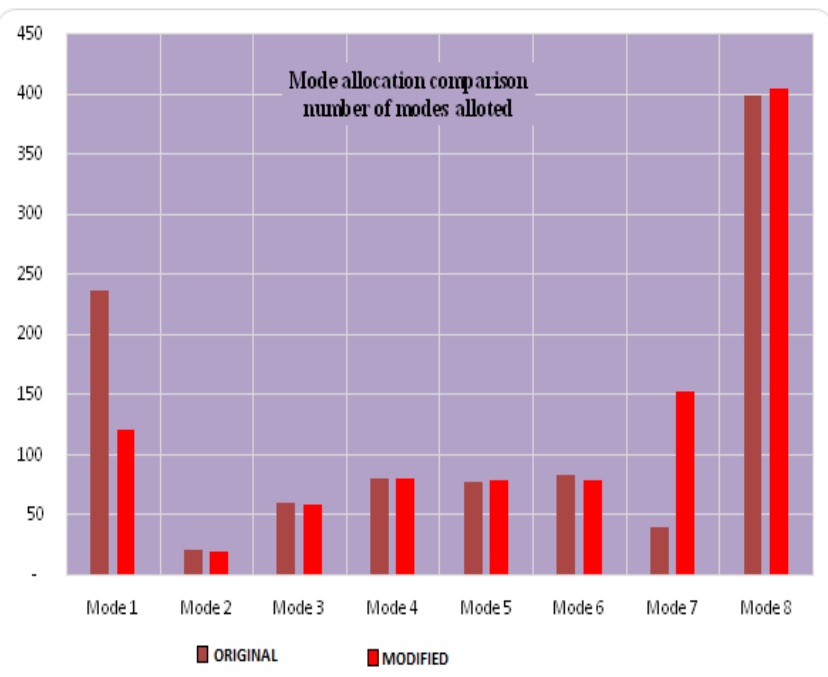

Fig.5. Mode allocation comparison Graph: Original v/s Modified Algorithm
The performance of both the algorithms was compared with the help of the graph shown in the figure no.5 above indicating that in the modified algorithm lowest mode gets selected less number of times compared to the originalone, while highest mode i.e. mode 8 gets selected higher number of times. The difference is appreciable if we see the frequency of selection of mode 1 and mode 7. It is obvious from the graph that, when modified algorithm was used frequency of occurrence of mode 1 is very low and that of mode7 is comparatively very high. Thus, it shows that higher modes are getting offered more frequently in the modified algorithm, as compared to the original algorithm.

\subsection{Comparison in terms of total bits transmitted per iteration}

As a result of higher number of modes being offered, the data rate improvement is expected and the same has been shown by calculating average data rates, corresponding to each mode allotted.

Table 4: Average Data Rate achieved: Original Algorithm v/s Modified Algorithm

\begin{tabular}{|l|l|l|}
\cline { 2 - 3 } \multicolumn{1}{c|}{} & \multicolumn{2}{c|}{ Average data rate achieved in each iteration } \\
\hline \multirow{2}{*}{} & $\begin{array}{l}\text { Original Algorithm } \\
\text { Data rate in MBPS }\end{array}$ & $\begin{array}{l}\text { Modified } \\
\text { Algorithm } \\
\text { Data rate in } \\
\text { MBPS }\end{array}$ \\
\hline 1 & 19.99 & 27.82 \\
\hline 2 & 19.04 & 27.32 \\
\hline 3 & 19.22 & 27.43 \\
\hline 4 & 20.27 & 28.07 \\
\hline 5 & 20.43 & 27.88 \\
\hline 6 & 18.98 & 27.85 \\
\hline 7 & 19.91 & 27.18 \\
\hline 8 & 19.68 & 28.37 \\
\hline 9 & 19.33 & 27.02 \\
\hline 10 & 20.36 & 27.17 \\
\hline $\begin{array}{l}\text { Average } \\
\text { data rate }\end{array}$ & $\mathbf{1 9 . 7 2}$ & $\mathbf{2 7 . 6 1}$ \\
\hline
\end{tabular}

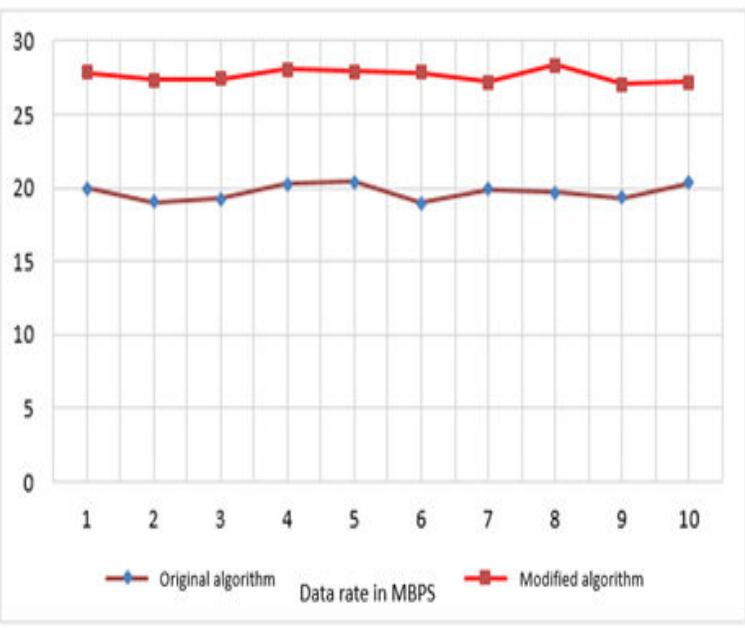

Fig.6. Average Data Rate achieved: Original Algorithm v/s Modified Algorithm 
The average data rates shown in table no.7 and graph in figure no. 19 are clearly indicating that the modified algorithm is able to enhance data rate i.e. from 19.72 MBPS to 27.61 MBPS (40\% increase).

\subsection{Comparison of the time to acquire a particular mode}

The following methodology was adapted to compare the time required to acquire a particular mode performance of the original and the modified algorithm.

1. Generate a set incremental SNR values (covering the minimum to maximum range of SNR) and feed that as input the algorithm

2. Observe and find out after how many number of steps next mode gets selected.

3. Note down the corresponding number of steps when all the eight modes get allotted as a result of linear increment of SNR.

4. Step a) to c) will form one complete iteration.

5. Repeat the iteration ( i.e. step a) to c)) for number of times.

6. Find out the cumulative value of number of turns needed when a particular mode is being allotted after completing all the iterations.

7. While analyzing and comparing the SNR value for which a particular mode gets allotted for the first time, the following results as mentioned in table no. 8 were obtained.

Table 5: Comparison of turns needed to achieve next higher mode

\begin{tabular}{|c|c|c|}
\hline & \multicolumn{2}{|c|}{ higher mode } \\
\hline & \multicolumn{2}{|c|}{ Cumulative value of minimum number of turns } \\
\hline & $\begin{array}{c}\text { Original } \\
\text { Algorithm }\end{array}$ & Modified Algorithm \\
\hline $\begin{array}{c}\text { Mode } \\
1-2\end{array}$ & 24.8 & 11.6 \\
\hline $\begin{array}{c}\text { Mode } \\
2-3\end{array}$ & 51.8 & 25.4 \\
\hline $\begin{array}{c}\text { Mode } \\
3-4\end{array}$ & 85.4 & 45.8 \\
\hline $\begin{array}{c}\text { Mode } \\
4-5\end{array}$ & 127.8 & 75 \\
\hline $\begin{array}{c}\text { Mode } \\
5-6\end{array}$ & 179 & 112.6 \\
\hline $\begin{array}{c}\text { Mode } \\
6-7\end{array}$ & 217 & 159.4 \\
\hline $\begin{array}{c}\text { Mode } \\
7-8\end{array}$ & 257.8 & 220.6 \\
\hline
\end{tabular}

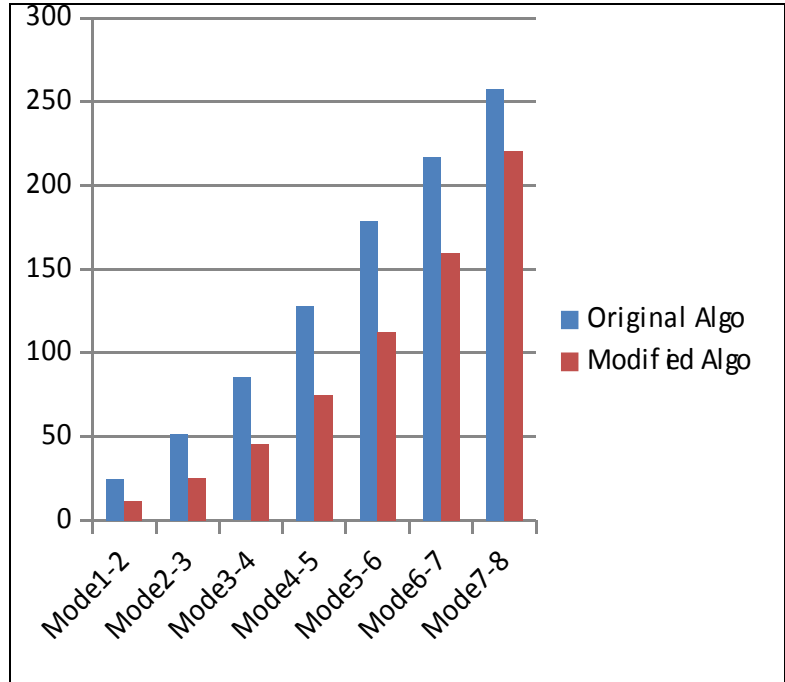

Fig.7. Cumulative value of turns required to achieve next higher mode

\section{CONCLUSION}

From the above discussion the following points are concluded:

We have seen that by estimating the channel condition with the help of its varying SNR value, the Adaptive Modulation and Coding scheme has been well implemented choosing higher order modulation and coding technique at high SNR values. Thus, this gave the higher bit rates, whereas choosing lower order modulation and coding schemes at lower SNR values for lower bit rates. Also, By modifying the algorithm we have shown through the results that for same values of SNR, we were able to achieve enhancement in the data rates and reduction in the number of transitions needed to achieve a particular mode in the AMC environment.

\section{REFERENCES}

[1] A. Zalonis, N. Miliou, I.Dagres, A.Polydoros, and H.Bogucka, April 2010, Trends in Adaptive Modulation and Coding, Advances in Electronics and Telecommunications.

[2] H. Jung, K. Cho, Y. Seok, T. Kwon and Y. Choi, RARA: Rate Adaptation Using Rate-adaptive Acknowledgment for IEEE 802.11 WLANs.

[3] Xi Chen, P. Gangwal, and D. Qiao, March 2012, RAM: Rate Adaptation in Mobile Environments, IEEE Transactions on Mobile Computing.

[4] H. Jung, T. Taekyoung Kwon, K. Cho, Y. Choi, 2011, REACT: Rate Adaptation using Coherence Time in 802.11 WLANs", Computer Communications.

[5] Y. Song, X. Zhu, Y. Fang, and H. Zhang, "Threshold Optimization for Rate Adaptation Algorithms in IEEE 802.11 WLANs", Ieee Transactions on Wireless Communications, VOL. 9, NO. 1, January 2010.

[6] K. M. Shaikh, "The Performance Evaluation of OFDM Based WLAN (IEEE 802.11a and 802.11g)", Master Thesis, Computer Science, December 2009.

[7] G. Holland, N. Vaidya and P. Bahl, "A Rate-Adaptive MAC Protocol for Multi-Hop Wireless Networks," in Proc. ACM SIGMOBILE’01, July 2001, pp. 236-251. 
[8] A. Hithnawi, "An On-Demand Rate-Adaptation Mechanism For IEEE 802.11 Networks" Master Thesis.

[9] M. Lacage, Mohammad H. Manshaei, and T. Turletti, "IEEE 802.11 Rate Adaptation: A Practical Approach, MSWiM'04, October 4-6,2004, Venezia, Italy.

[10] C. Cheny, E. Seoy, H. Luoy, and N. H. Vaidya, "Rate adaptive Framing for Interfered Wireless Networks", Technical Report UIUCDCSR20062743.

[11] B. Sadeghi, V. Kanodia, A. Sabharwal, and E. Knightly,"OAR: an opportunistic auto-rate media access protocol for ad hoc networks" Wireless Networks, vol. 11, pp. 39-53, 2005.

[12] Jiansong Zhang Kun Tan Jun Zhao Haitao Wu Yongguang Zhang "A practical SNR guided Rate adaptation", IEEE INFOCOM 2008 proceedings.

[13] J. P. Pavon and C. Sunghyun,"Link adaptation strategy for IEEE 802.11 WLAN via received signal strength measurement" in 2003 IEEE International Conference on Communications (Cat. No.03CH37441), 2003, pp.110813 vol.2.

[14] IEEE Std 802.11a-1999 (Supplement to IEEE Std 802.11-1999), Part 11: Wireless LAN Medium Access Control (MAC) and Physical Layer (PHY) specifications: High-speed Physical Layer in the $5 \mathrm{GHZ}$ Band.
[15] A. Goldsmith and S.G. Chua "Variable-rate variablepower MQAM for fading channels." IEEE Trans. Commun., pp. 1218-1230, Oct. 1997.

[16] Link adaptation for IEEE 802.11a WLAN over fading channel Department of Communication Technology, Aalborg University, January 2nd,2004.

[17] John C.Bicket, "Bit-rate Selection in Wireless Network", Master Thesis, Computer Science and Engineering, Massachusetts Institute of Technology, February 2005

[18] L. Caponi, F. Chiti, and R. Fantacci, "Performance Evaluation of a Link Adaptation Technique for High Speed Wireless Communication Systems," IEEE Transactions on Wireless Communications, VOL. 6, No. 12, December 2007.

[19] S. T. Chung, and A. J. Goldsmith, "Degrees of Freedom in Adaptive Modulation: A Unified View," Ieee Transactions on Communications, VOL. 49, NO. 9, September 2001.

[20] A. Muller, P. Frank, "Cooperative Interference Prediction for Enhanced Link Adaptation in the 3GPP LTE Uplink,"

[21] "Channel Estimation and Modelling" S-72.333 Postgraduate Course in Radiocommunications, 2000.

[22] "Channel prediction for adaptive modulation in wireless communications" thesis by R. Chan, Blacksburg, Virginia,

July 16 , 2003. 